\title{
ASSESSMENT OF COMPLEX VARIABLE BASIS FUNCTIONS IN THE APPROXIMATION OF IDEAL FLUID FLOW PROBLEMS
}

\author{
BRYCE D. WILKINS, T.V. HROMADKA II, ANTHONY N. JOHNSON, RANDY BOUCHER, \\ HOWARD D. MCINVALE \& STEVE HORTON \\ Department of Mathematical Sciences, United States Military Academy, USA.
}

\begin{abstract}
Solving potential problems, such as those that occur in the analysis of steady-state heat transfer, electrostatics, ideal fluid flow, and groundwater flow, is important in several fields of engineering, science, and applied mathematics. Numerical solution of the relevant governing equations typically involves using techniques such as domain methods (including finite element, finite difference, or finite volume), or boundary element methods (using either real or complex variables). In this paper, the Complex Variable Boundary Element method ("CVBEM") is examined with respect to the use of different types of basis functions in the CVBEM approximation function. Four basis function families are assessed in their solution success in modeling an important benchmark problem in ideal fluid flow; namely, flow around a 90 degree bend. Identical problem domains are used in the examination, and identical degrees of freedom are used in the CVBEM approximation functions. Further, a new computational modeling error is defined and used to compare the results herein; specifically, $M=E / N$ where $M$ is the proposed computational error measure, $E$ is the maximum difference (in absolute value) between approximation and boundary condition value, and $N$ is the number of degrees of freedom used in the approximation. Keywords: basis functions, Complex Variable Boundary Element Method (CVBEM), complex variables, computational fluid dynamics (CFD), flow nets, ideal fluid flow, Laplace equation
\end{abstract}

\section{INTRODUCTION}

The Complex Variable Boundary Element Method (CVBEM) is the topic of many papers and books [1-7]. Further, although the theory of complex variables, and the applications thereof, are typically limited to two dimensions, the CVBEM type of approximation has successfully been applied to three and higher spatial dimensions; for example, on a sphere in $\mathrm{R}^{3}$. For details regarding the development of CVBEM type approximation functions in three and higher dimensions see Hromadka [8].

The original CVBEM basis functions were derived by using polynomial interpolation of given potential and streamline values along the problem boundary to generate an approximate boundary. If linear trial functions are used in the interpolation, then the CVBEM basis functions that result from numerically solving the Cauchy integral are products of complex linear polynomials with complex logarithmic functions. Likewise, when higher-order polynomial trial functions are used in the interpolation of the problem boundary, the resulting CVBEM approximation function is similarly composed of basis functions that are products of complex polynomials with complex logarithmic functions. However, while the use of higher-order polynomial trial functions in the interpolation of the problem boundary is possible, in this research, the basis functions resulting from linear interpolation along the problem boundary are used.

It is noted that the term CVBEM refers to the general use of complex basis functions to solve boundary value problems, provided that the basis functions are analytic within the problem domain. Further, it is possible to mix families of basis functions to better fit particular problem geometries, although, such hybridizations are not considered in this research. 
This research examines the solution success of families of basis functions in addition to those derived from the numerical solution of the Cauchy integral. All of the CVBEM approximation functions considered have the desirable property that they are well-defined and analytic throughout the problem domain as well as along the approximate boundary that is being modeled, see Johnson, et al. [9]. A CVBEM approximation function is a linear combination of members of the basis function family being used in the approximation. Since the basis functions examined in this research are analytic in the problem domain and along the boundary, the CVBEM approximation function is itself analytic in that region. There are two properties that follow directly from the fact that CVBEM approximation functions are analytic within the problem domain and along the approximate boundary:

1. CVBEM approximation functions are capable of providing computational estimates continuously throughout the problem domain and approximate boundary,

2. and, both the real and imaginary parts of a CVBEM approximation function exactly solve the two-dimensional Laplace equation, as well as similar PDEs such as the Poisson equation, throughout the problem domain.

These properties of CVBEM approximation functions are not shared with real-variable domain discretization techniques such as the finite element (FEM) and finite difference methods (FDM), which develop point estimates at nodal points defined by meshing the problem domain. However, both the real and imaginary components of the approximation function resulting from the CVBEM do exactly solve Laplace's equation in its strong formulation.

Another advantage afforded by the use of CVBEM approximation functions is that both the real and imaginary parts are conjugate functions. So, when solving potential problems such as ideal fluid flow problems, the resulting imaginary part of the CVBEM approximation function is the streamline function whose isocontours are orthogonal to the isocountours of the potential function (the real part) and, when plotted, produce the standard flow net graphical display, see Johnson et al. [10].

Thus, given potential boundary values, when using the CVBEM to generate flow net displays, it is not necessary to use another software program, or another post-processing routine, to generate orthogonal streamlines to the calculated potential lines. Rather, the orthogonal isocontours associated with flow nets are a direct outcome of the CVBEM itself. Further, these streamline functions are analytic at all points at which the potential function is analytic. So, the streamline function can similarly be evaluated at all points in the problem domain. In comparison, the FDM and FEM approximations require another analog to estimate streamlines using the point set of potential function estimates developed therefrom.

In all cases tested, modeling nodes and collocation points are positioned identically. To compare modeling error, a computational measure is used defined by $M=E / N$, where $M$ is the proposed computational error measure value, $\mathrm{E}$ is the maximum departure between approximation and boundary condition value along the problem boundary (in absolute value), and $N$ is the number of degrees of freedom used in the approximation. Further, flow nets are developed for illustration and comparison purposes.

In the diagrams provided, there are no nodes or computational grid type points used or shown in the interior of the problem domain, and no companion interpolation scheme or computer program is used in the development of the displayed flow net diagrams. Rather, the values of the potential and streamline functions, which are used to generate the accompanying flow net diagrams, are developed by evaluating the CVBEM approximation function for 
both the potential and streamline functions throughout the interior of the problem domain. It is noted that comparisons of this type have been done before, for example, see Barone et al. [11]. However, not only does this research demonstrate the solution success of new basis function families (those of eqns (4) and (5) below), this analysis is more comprehensive than previous research because it includes an analysis of the convergence of each basis function family for various degrees of freedom.

\section{REVIEW OF THE CVBEM MODELING APPROACH}

A CVBEM approximation function, $\hat{\omega}$, has the general form

$$
\hat{\omega}(z)=\sum_{j=1}^{n} \mathrm{C}_{j} g_{j}(z),
$$

where $\mathrm{C}_{j}$ is the $j^{\text {th }}$ complex coefficient, $g_{j}(\mathrm{z})$ is the $j^{\text {th }}$ member of the family of basis functions being used in the approximation, and $\mathrm{n}$ is the number of basis functions being used in the approximation. The complex coefficients are composed of two real constants; the real and the imaginary components, both coefficients are degrees of freedom to be determined as part of the CVBEM modeling process. Thus, there are $2 n$ degrees of freedom in a CVBEM approximation function. Various techniques for determining these CVBEM coefficients are examined in the literature, including Fredholm integral type of formulations, and minimization of departure between boundary condition values and CVBEM boundary values with respect to least squares minimization or by collocation, among other techniques, see Bohannon [12]. In this paper, collocation is used to determine coefficient values.

The error of the CVBEM approximation function can be determined by considering the difference between the CVBEM approximation function and the analytic potential solution (eqn (6) below). The difference between these two functions is the "error" function, and since it is the difference of two analytic functions over the problem domain and approximate boundary, it is itself analytic over that region. Thus, the error function attains its maximum (and minimum) values on the problem boundary by the Maximum Modulus Theorem. Therefore, in the case of a Dirichlet boundary value problem, modeling error in the approximation potential function can be assessed by examination of the maximum departure between the boundary values of the CVBEM approximation potential function and the boundary values of the analytic potential solution. It is noted that modeling error can be reduced by inserting additional collocation points in the vicinity of areas of large modeling error values.

The fundamental goal of modeling with the CVBEM is to produce an approximation function that is analytic over the entire problem domain and its boundary (provided that modeling nodes are located exterior to the domain union boundary). It is noted that the choice of the analytic basis functions to be used in the prescribed linear combination of eqn (1) may be motivated by the problem boundary geometry in order to simplify the modeling requirements and to better fit the boundary geometry.

Because the CVBEM approach yields an approximation function that is analytic over the entire problem domain, and along the approximate boundary for properly-formulated models, the real and imaginary component functions are both well-defined functions over the entire domain and boundary (and, therefore, do not require employment of interpolation functions to estimate modeling values of either function in the interior of the problem domain or on the problem boundary). Further, both the real and imaginary component functions of the CVBEM approximation function exactly satisfy the two-dimensional Laplace equation 
continuously over the entire problem domain and on the problem boundary. This property enables considerable advantages over discrete domain methods such as FEM and FDM, for example, that estimate point values at discrete locations throughout the interior of the problem domain and do not satisfy, but only approximately solve, the Laplace equation (or other target PDE).

The four basis function families considered are:

1. products of complex linear polynomials and complex logarithms with properly-rotated branch cuts so that the branch associated with each logarithm lies outside of the problem domain (these follow from numerically solving the Cauchy integral);

$$
\left(z-z_{j}\right) \ln \left(z-z_{j}\right)
$$

2. complex monomials (known in the literature as the Complex Variable Polynomial Method or "CVPM"), which is similar in principle to a Taylor series approximation and evaluated about a target expansion point, $\mathrm{z}_{0}$;

$$
\left(z-z_{0}\right)^{j}
$$

3. a Laurent series expansion (reciprocals of complex monomials evaluated at a target expansion point, $\mathrm{z}_{1}$ );

$$
\frac{1}{\left(z-z_{1}\right)^{j}}
$$

4. and, the sum of reciprocals of complex linear polynomials evaluated at a set of nodal points (positioned identically to the nodes used in the implementation of the CVBEM using the basis functions of eqn (2))

$$
\frac{1}{z-z_{j}}
$$

In eqns (2)-(5), $z$ is a complex variable, $z_{j}$ is a node, and $z_{0}$ and $z_{1}$ are points in the complex plane that are located exterior to the problem domain union boundary. The nodes that are used in eqns (2) and (5) are placed identically.

\section{APPLICATION AND COMPARISON OF FOUR CVBEM BASIS FUNCTION FAMILIES}

The four basis function families herein considered are eqns (2)-(5). The choice of these basis function families was arbitrary with the condition that the basis functions must be analytic over at least the domain of interest. However, the selections that were made follow the construct seen in the commonly used series expansions such as linear interpolation in numerical approximation of the Cauchy Integral, Taylor series, Laurent Series, and distributed source terms (in polar coordinates). Other basis function families are readily available, and it is noted that the choice of basis function family can lead to better approximation performance for some types of boundary value problems and poorer approximation performance for other 
problems. Nonetheless, regardless of basis function family type, the resulting approximations are analytic over the problem domain and, hence, all have the various properties associated with analytic functions.

The point $z_{0}=20+20 i$ is used as the point of expansion for the complex monomials of eqn (3), and the point $z_{1}=20+20 i$ is used as the point of expansion for the Laurent type of basis functions of eqn (4). As done in Johnson et al. [13], the modeling nodes used in eqns (2) and (5) are specified exterior to the problem domain union boundary (shown in the figures of Section 4).

The problem under consideration is modeling the flow of an ideal fluid around a 90 degree bend. The analytic solution describing such ideal fluid flow is the complex-valued function

$$
f(z)=z^{2}
$$

The problem domain is a rectangle with corners located at $(0,0),(2,0),(2,1)$, and $(0,1)$. For the test problem, the monomial $\left(z-z_{0}\right)^{2}$ is eliminated from the basis of eqn (3). Collocation points were spaced evenly along the boundary with the first collocation point being located at the origin and the other collocation points being placed equidistant around the boundary in a counterclockwise direction. Model nodes are positioned equally spaced along a circle of radius 1.8 that was centered at the center of the rectangle $(1,0.5)$. All nodes are located exterior to the problem domain union boundary. Additionally, when the CVBEM basis functions of eqn (2) were used, the respective branch cuts were rotated so as to be directed 180 degrees away from the directed line segment from each node to the center of the rectangular domain. The first node was located at $(-0.8,0.5)$ and all subsequent nodes were equally spaced along the described circle.

For each family of basis functions, the CVBEM approximation functions were generated by using only the position and value of each collocation point, as well as the position and branch cut rotation of each node for the basis function families of eqns (2) and (5). As with any technique for solving boundary value problems, the CVBEM operates only on the boundary values of the problem. In this research, only boundary values from the potential function were used as the collocation points. So, the streamlines in Section 4 were generated without collocating the CVBEM approximation function with any streamline boundary values. That is, they are a direct product of the CVBEM technique itself. The ability to accurately approximate streamlines is essential to modeling ideal fluid flow.

The CVBEM approximation functions used in this research were obtained in the following way. By eqn (1), CVBEM approximation functions have the form

$$
\hat{\omega}(z)=\mathrm{C}_{1} g_{1}(z)+\mathrm{C}_{2} g_{2}(z)+\cdots+\mathrm{C}_{n} g_{n}(z)
$$

Further, since each $\mathrm{C}_{\mathrm{j}}$ is a complex constant, it has a real and an imaginary part, $\alpha_{j}$ and $\beta_{j}$, respectfully. Thus, the general CVBEM approximation function is

$$
\hat{\omega}(z)=\left(\alpha_{1}+i \beta_{1}\right) g_{1}(z)+\left(\alpha_{2}+i \beta_{2}\right) g_{2}(z)+\cdots+\left(\alpha_{n}+i \beta_{n}\right) g_{n}(z) .
$$

Letting $\lambda_{j}(z)$ and $\mu_{j}(z)$ represent the real and imaginary component functions, respectively, of $g_{j}(\mathrm{z})$, then

$$
\hat{\omega}(z)=\left(\alpha_{1}+i \beta_{1}\right)\left(\lambda_{1}(z)+i \mu_{1}(z)\right)+\left(\alpha_{2}+i \beta_{2}\right)\left(\lambda_{2}(z)+i \mu_{2}(z)\right)+\cdots+\left(\alpha_{n}+i \beta_{n}\right)\left(\lambda_{n}(z)+i \mu_{n}(z)\right) .
$$


Thus the real part $\hat{\phi}$ of the CVBEM approximation function $\hat{\omega}$, which represents the potential function, is

$$
\hat{\phi}(z)=\alpha_{1} \lambda_{1}(z)-\beta_{1} \mu_{1}(z)+\alpha_{2} \lambda_{2}(z)-\beta_{2} \mu_{2}(z)+\cdots+\alpha_{n} \lambda_{n}(z)-\beta_{n} \mu_{n}(z)
$$

Likewise, the imaginary part, $\hat{\psi}$, of the CVBEM approximation function, $\hat{\omega}$, which represents the streamline function is

$$
\hat{\psi}(z)=\alpha_{1} \mu_{1}(z)+\beta_{1} \lambda_{1}(z)+\alpha_{2} \mu_{2}(z)+\beta_{2} \lambda_{2}(z)+\cdots+\alpha_{n} \mu_{n}(z)+\beta_{n} \lambda_{n}(z) .
$$

In this research, boundary conditions of the potential function are specified at the collocation points. Thus, for $2 n$ collocation points, the necessary CVBEM approximation functions are

$$
\begin{aligned}
& \hat{\phi}\left(z_{1}\right)=\alpha_{1} \lambda_{1}\left(z_{1}\right)-\beta_{1} \mu_{1}\left(z_{1}\right)+a_{2} \lambda_{2}\left(z_{1}\right)-\beta_{2} \mu_{2}\left(z_{1}\right)+\cdots+a_{n} \lambda_{n}\left(z_{1}\right)-\beta_{n} \mu_{n}\left(z_{1}\right) \\
& \hat{\phi}\left(z_{2}\right)=\alpha_{1} \lambda_{1}\left(z_{2}\right)-\beta_{1} \mu_{1}\left(z_{2}\right)+a_{2} \lambda_{2}\left(z_{2}\right)-\beta_{2} \mu_{2}\left(z_{2}\right)+\cdots+a_{n} \lambda_{n}\left(z_{2}\right)-\beta_{n} \mu_{n}\left(z_{2}\right) \\
& \vdots \\
& \hat{\phi}\left(z_{2 n}\right)=\alpha_{1} \lambda_{1}\left(z_{2 n}\right)-\beta_{1} \mu_{1}\left(z_{2 n}\right)+\alpha_{2} \lambda_{2}\left(z_{2 n}\right)-\beta_{2} \mu_{2}\left(z_{2 n}\right)+\cdots+a_{n} \lambda_{n}\left(z_{2 n}\right)-\beta_{n} \mu_{n}\left(z_{2 n}\right)
\end{aligned}
$$

This yields the following matrix equation.

$$
\left[\begin{array}{l}
\hat{\phi}\left(z_{1}\right) \\
\hat{\phi}\left(z_{2}\right) \\
\vdots \\
\hat{\phi}\left(z_{2 n}\right)
\end{array}\right]=\left[\begin{array}{ccccccc}
\lambda_{1}\left(z_{1}\right) & -\mu_{1}\left(z_{1}\right) & \lambda_{2}\left(z_{1}\right) & -\mu_{2}\left(z_{1}\right) & \cdots & \lambda_{n}\left(z_{1}\right) & -\mu_{n}\left(z_{1}\right) \\
\lambda_{1}\left(z_{2}\right) & -\mu_{1}\left(z_{2}\right) & \lambda_{2}\left(z_{2}\right) & -\mu_{2}\left(z_{2}\right) & \cdots & \lambda_{n}\left(z_{2}\right) & -\mu_{n}\left(z_{2}\right) \\
& & & \vdots & & & \\
\lambda_{1}\left(z_{2 n}\right) & -\mu_{1}\left(z_{2 n}\right) & \lambda_{2}\left(z_{2 n}\right) & -\mu_{2}\left(z_{2 n}\right) & \cdots & \lambda_{n}\left(z_{2 n}\right) & -\mu_{n}\left(z_{2 n}\right)
\end{array}\right]\left[\begin{array}{l}
\alpha_{1} \\
\beta_{1} \\
\alpha_{2} \\
\beta_{2} \\
\vdots \\
\alpha_{n} \\
\beta_{n}
\end{array}\right]
$$

The final step is to solve the matrix system. Once these values are known, they can be substituted back into eqn (7) and can then be used to approximate all of the potential values within the problem domain. Likewise, these values can be substituted back into eqn (8) and can be used to approximate all of the streamline values within the problem domain. Note that it is possible to approximate all of the streamline values within the problem domain without knowing any streamline boundary conditions. That is, the equation for the streamline function is a direct product of the CVBEM.

From the Maximum Modulus Theorem, the maximum error of the CVBEM approximation function occurs on the boundary. Therefore, in order to determine the maximum error, the CVBEM approximation potential function was evaluated at 600 points along the boundary and the value of the CVBEM approximation potential function at each point was compared to the analytic potential solution given by $f(x, y)=x^{2}-y^{2}$. The maximum error corresponded to the maximum departure (in absolute value) of the CVBEM approximation of the potential 
function from the analytic potential solution. The 600 test points were spaced evenly along the boundary with 200 test points located along the long ends of the rectangular boundary and 100 test points located along each of the short ends of the rectangular boundary.

\section{RESULTS}

In all of the figures below, the plot labeled (a) shows the complete problem domain as well as the location of all modeling nodes and collocation points. The nodes are represented by black circles, and the collocation points are shown as red circles. The problem boundary is outlined by a white rectangle. Additionally, for the basis functions of eqn (2), the white lines extending from the nodes represent the branch cuts of the complex logarithms (rotated as described above). The plot labeled (b) shows the approximate solution, and, importantly, the potential and streamline isocontours. These plots demonstrate the approximation generated by each basis function in modeling ideal fluid flow around a 90 degree bend.

\subsection{Numerical Results}

In the following table, each of the error values are calculated using the new error measure defined by $M=E / N$.

Table 1: Error Values for Each of the Families of Basis Functions

\begin{tabular}{lllll}
\hline & $\begin{array}{l}\text { Error for Original } \\
\text { CVBEM Basis }\end{array}$ & $\begin{array}{l}\text { Error for Complex } \\
\text { Monomials eqn (3) }\end{array}$ & $\begin{array}{l}\text { Error for Laurent } \\
\text { Series eqn (4) }\end{array}$ & $\begin{array}{l}\text { Error for Singularity } \\
\text { Basis Functions } \\
\text { eqn (5) }\end{array}$ \\
\hline $4.0 . f$. & Functions eqn (2) & (2.8033e-03 & $4.7539 \mathrm{e}-02$ & $5.7742 \mathrm{e}-01$ \\
8 & $2.1704 \mathrm{e}-01$ & $2.1973 \mathrm{e}-06$ & $8.9483 \mathrm{e}-05$ & $8.5576 \mathrm{e}-03$ \\
16 & $4.9659 \mathrm{e}-03$ & $8.2509 \mathrm{e}-13$ & $2.9467 \mathrm{e}-10$ & $3.2290 \mathrm{e}-03$ \\
32 & $5.4397 \mathrm{e}-05$ & $4.6615 \mathrm{e}-14$ & $7.8320 \mathrm{e}-12$ & $2.9537 \mathrm{e}-05$ \\
64 & $1.4154 \mathrm{e}-07$ & $9.0428 \mathrm{e}-14$ & $6.4503 \mathrm{e}-12$ & $1.4917 \mathrm{e}-09$ \\
128 & $2.0602 \mathrm{e}-12$ & $6.9337 \mathrm{e}-14$ & $3.5780 \mathrm{e}-13$ & $2.8271 \mathrm{e}-15$ \\
\hline
\end{tabular}

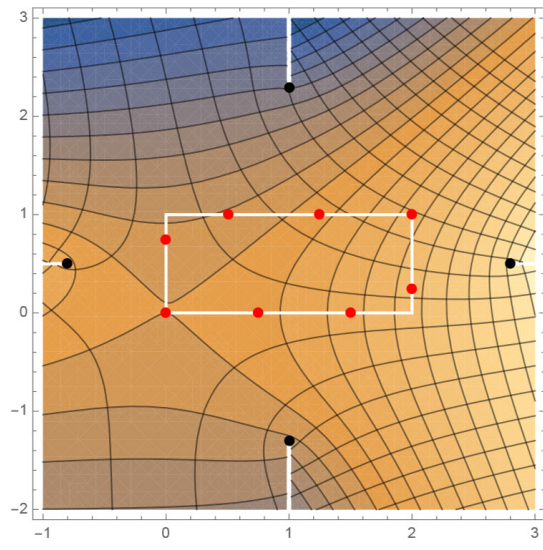

(a) Domain and Node Locations

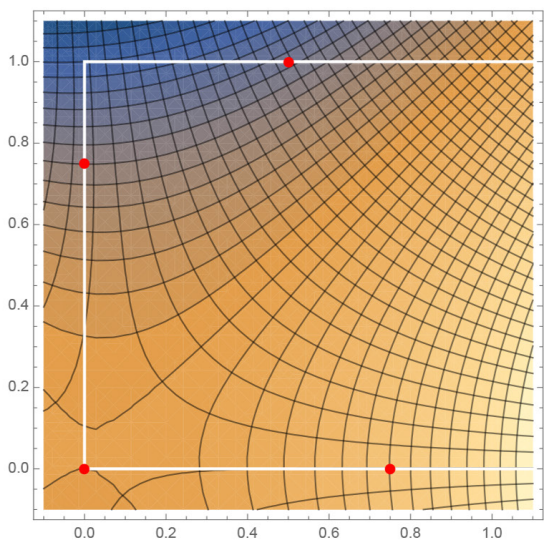

(b) Approximation of ideal fluid flow.

Figure 1: Products of complex Linear Polynomials and Complex Logarithms (8 d.o.f) 


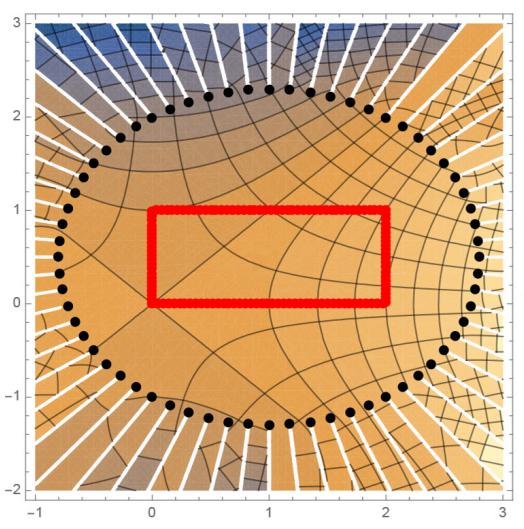

(a) Domain and Node Locations

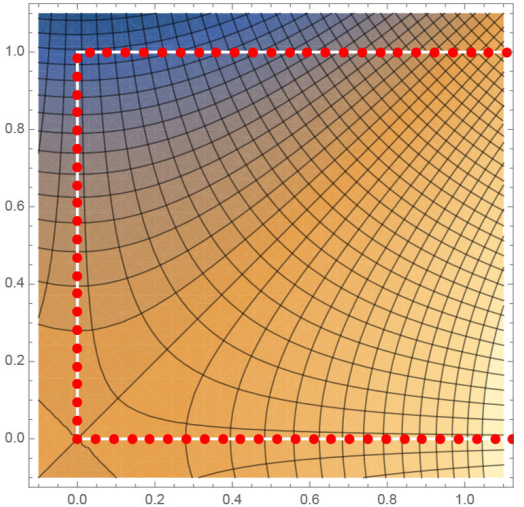

(b) Approximation

Figure 2: Products of Complex Linear Polynomials and Complex Logarithms (128 d.o.f.)

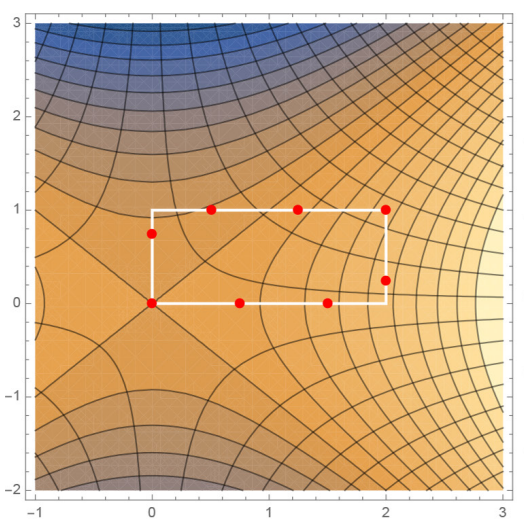

(a) Domain and Node Locations

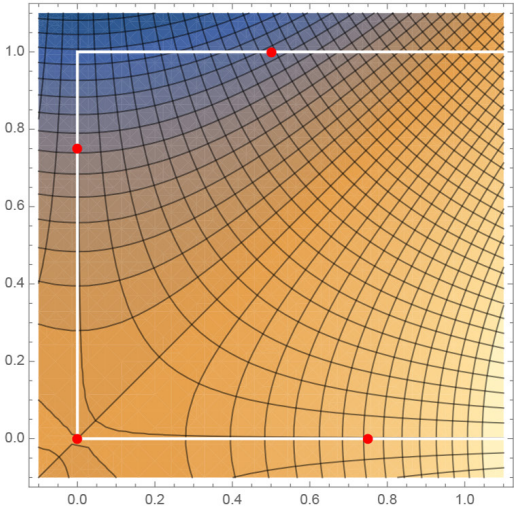

(b) Approximation

Figure 3: Complex Monomials - Taylor Series Analogy (8 d.o.f.)

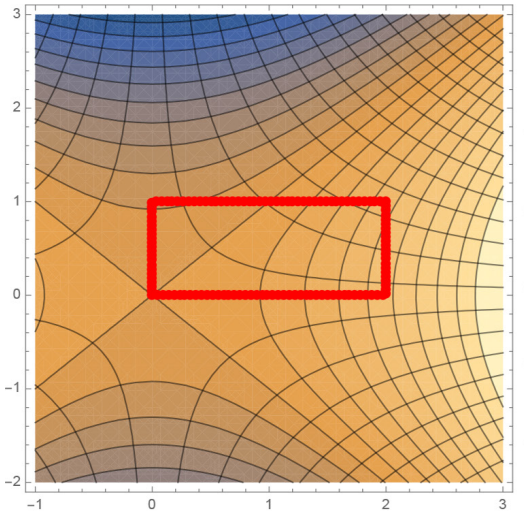

(a) Domain and Node Locations

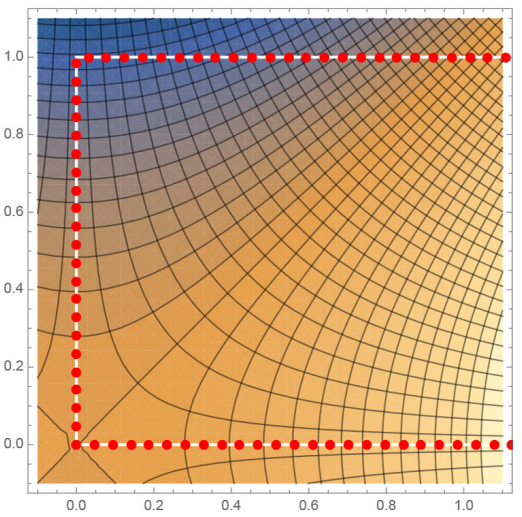

(b) Approximation

Figure 4: Complex Monomials - Taylor Series Analogy (128 d.o.f.) 


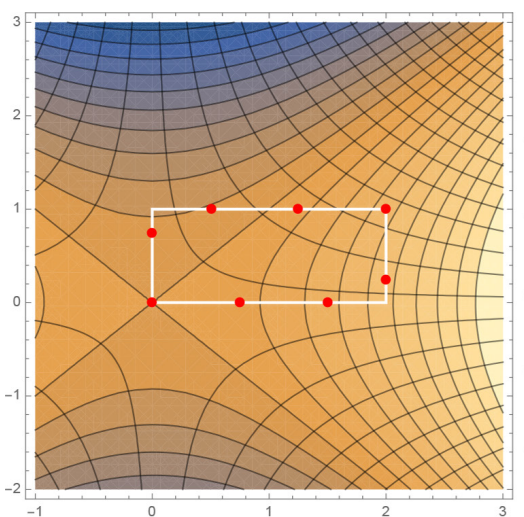

(a) Domain and Node Locations

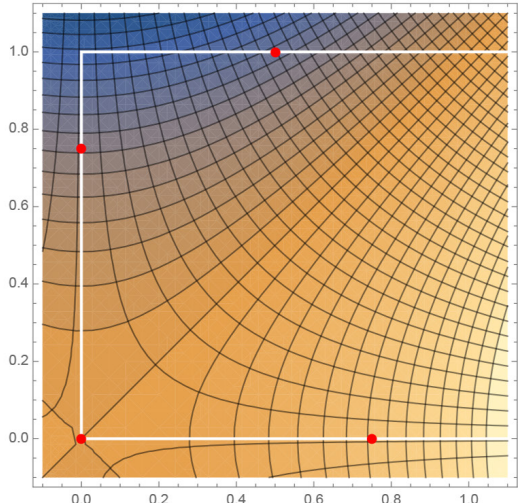

(b) Approximation

Figure 5: Reciprocals of Complex Monomials - Laurent Series Analogy (8 d.o.f.)

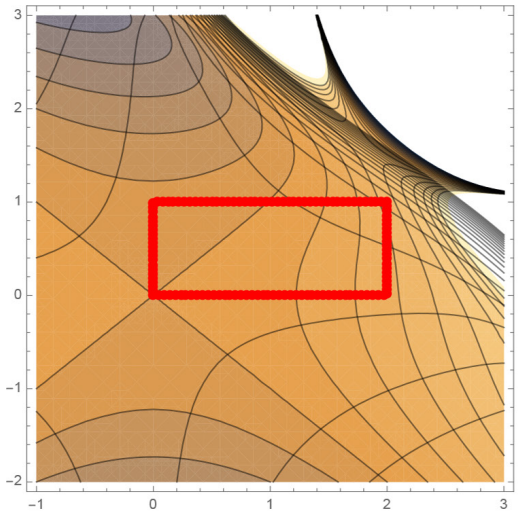

(a) Domain and Node Locations

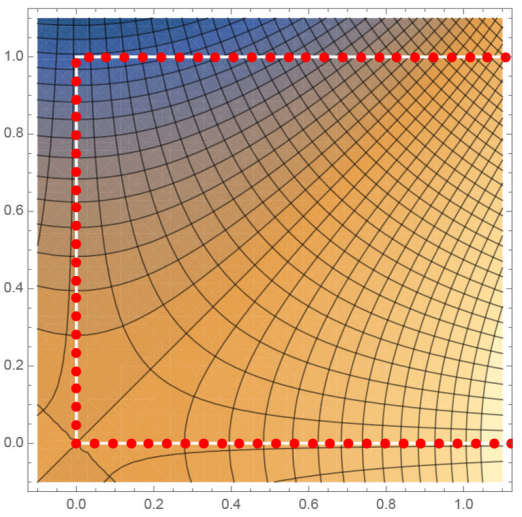

(b) Approximation

Figure 6: Reciprocals of Complex Monomials - Laurent Series Analogy (128 d.o.f.)

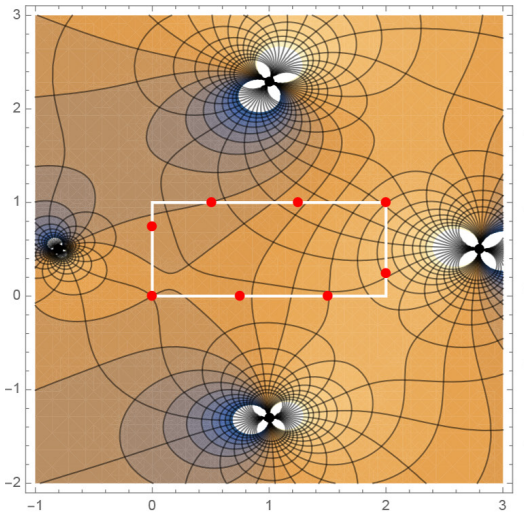

(a) Domain and Node Locations

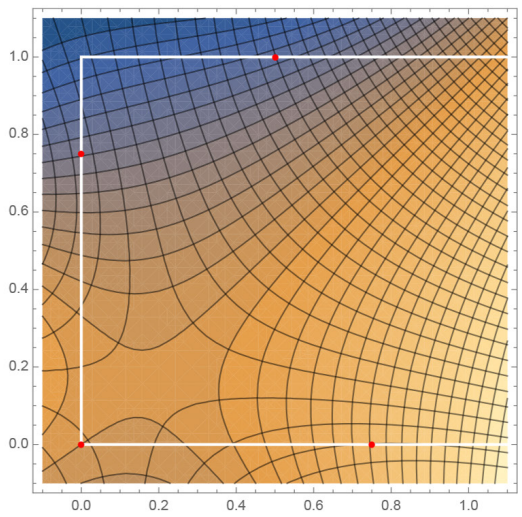

(b) Approximation

Figure 7: Reciprocals of Complex Linear Polynomials (8 d.o.f.) 


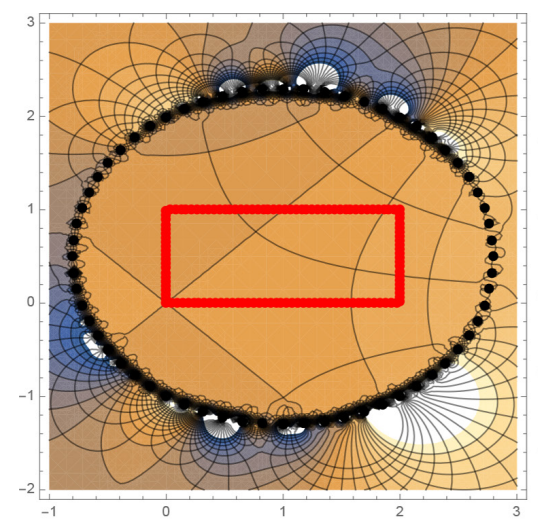

(a) Domain and Node Locations

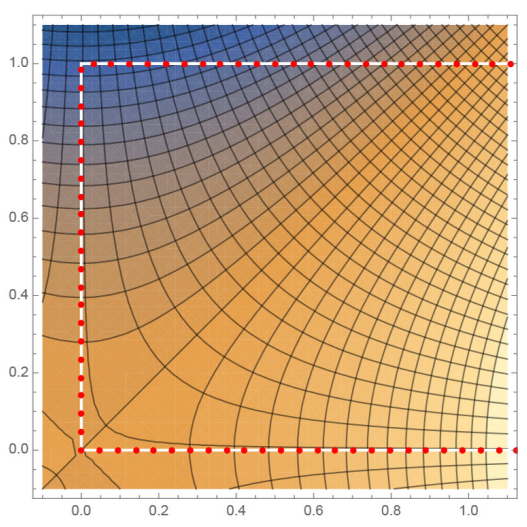

(b) Approximation

Figure 8: Reciprocals of Complex Linear Polynomials (128 d.o.f.)

\section{DISCUSSION OF COMPUTATIONAL OUTCOMES}

The figures display the flow nets produced by the CVBEM for each of the four basis functions examined. In all four cases, the identical problem geometry and boundary conditions are imposed for solution of the governing PDE; namely, the Laplace equation. The same number of modeling degrees of freedom are involved for each of the four approximation efforts. The locations of nodes and collocation point are all identically specified and evenly spaced along the problem boundary. The computational error, $M=E / N$ (as defined previously) provides a convenient measure to compare computational results and accuracy for the various degrees of freedom used in each approximation. The tabulation of Table 1 compares the computational effectiveness in using the four considered basis function families.

The computational outcomes depend on the distribution of modeling nodes and collocation point locations as well as the locations of the expansion points for the Taylor Series (CVPM) and Laurent Series type of basis function approximations. Multiple source points for the Taylor and Laurent Series can be considered as well. Further, considering the possibility of producing CVBEM hybridizations by mixing basis functions, more research is needed to assess whether introducing different types of basis functions provides computational advantage over using higher dimension in any one particular basis function family. For example, in a Taylor Series type of approximation, it is not clear whether inclusion of higher degree monomials is preferable over the introduction of another type of basis function such as a complex logarithm basis function or other type.

\section{CONCLUSIONS}

Recently, the Complex Variable Boundary Element Method or "CVBEM" has been the subject of several threads of computational research leading to multiple advances including, but not limited to: relaxing the usual approach of locating model nodal points on the problem boundary and, instead, placing the nodes in the exterior of the domain union boundary; using products of higher degree complex polynomials and complex logarithms as basis functions in the CVBEM approximation, as well as using different basis function families; and, evaluating different methods of minimizing numerical error in fitting problem boundary conditions 
such as collocation and least squares minimization. In this paper, we assess the advantages afforded by the four different families of basis functions in eqns (2)-(5) as to their possible success in modeling an important two-dimensional ideal fluid flow problem: namely, flow around a 90 degree bend.

The four families are applied to the identical ideal fluid flow problem setting using collocation to determine the necessary complex constant coefficient values. In all cases tested, nodes and collocation points are positioned identically. To compare modeling error, a new computational measure is applied defined by $M=E / N$, where $M$ is the computational measure value, $E$ is the maximum computational departure between approximation and boundary condition value along the entire problem boundary (in absolute value), and $N$ is the number of degrees of freedom used in the approximation (equivalently, the number of real-valued co-efficients determined in the CVBEM modeling process for use in the approximation function linear combination.) Flow nets are developed for graphical comparison purposes. Computational results indicate that choice of basis function family primarily impacts the rapidity of convergence of the approximation function.

\section{ACKNOWLEDGEMENTS}

The authors acknowledge the Department of Mathematical Sciences at the United States Military Academy, West Point, New York, the United States Army, and the many individuals who have participated and contributed to CVBEM research. An assembly of these works can be found at www.USMAthematics.com.

\section{REFERENCES}

[1] Hromadka, T.V. \& Lai, C., The Complex Variable Boundary Element Method. SpringerVerlag, New York, 1987.

[2] Hromadka, T.V. \& Whitley, R.J., Approximating three-dimensional steady-state potential flow problems using two-dimensional complex polynomials. Engineering Analysis with Boundary Elements, 29, pp. 190-194, 2005.

https://doi.org/10.1016/j.enganabound.2004.07.004

[3] Hromadka, T.V. \& Whitley, R., Foundations of the Complex Variable Boundary Element Method. Springer, Berlin, 2014.

[4] Hromadka, T.V. \& Whitley, R.J., Advances in the Complex Variable Boundary Element Method. Springer, New York, 1998.

[5] Hromadka, T. \& Whitley, R., A new formulation for developing CVBEM approximation functions. Engineering Analysis with Boundary Elements, 18(1), pp. 39-41, 1996.

https://doi.org/10.1016/s0955-7997(96)00027-6

[6] Dumir, P. \& Kumar, R., Complex variable boundary element method for torsion of anisotropic bars. Applied Mathematical Modelling, 17(2), pp. 80-88, 1993. https://doi.org/10.1016/0307-904x(93)90096-y

[7] Hromadka, T.V. \& Pardoen, G.C., Application of the CVBEM to non-uniform st. Venant torsion. Computer Methods in Applied Mechanics and Engineering, 53(2), pp. 149-161, 1985.

https://doi.org/10.1016/0045-7825(85)90003-9

[8] Hromadka, T.V., A Multi-Dimensional Complex Variable Boundary Element Method, volume 40 of Topics in Engineering. WIT Press, Southampton and Boston, 2002. 
[9] Johnson, A.N., Hromadka, T.V., Carroll, M., Hughes, M., Jones, L., Pappas, N., Thomasy, C., Horton, S., Whitley, R. \& Johnson, M., A computational approach to determining CVBEM approximate boundaries. Engineering Analysis with Boundary Elements, 41, pp. 83-89, 2014.

https://doi.org/10.1016/j.enganabound.2013.12.011

[10] Johnson, A.N., Hromadka, T.V., Hughes, M.T. \& Horton, S.B., Modeling mixed boundary problems with the complex variable boundary element method (CVBEM) using matlab and mathematica. International Journal of Computational Methods and Experimental Measurements, 3(3), pp. 269-278, 2015. https://doi.org/10.2495/cmem-v3-n3-269-278

[11] Barone, G., Pirrotta, A. \& Santoro, R., Comparison among three boundary element methods for torsion problems: CPM, CVBEM, LEM. Engineering Analysis with Boundary Elements, 35(7), pp. 895-907, 2011. https://doi.org/10.1016/j.enganabound.2011.02.003

[12] Bohannon, A. \& Hromadka, T., The complex polynomial method with a least-squares fit to boundary conditions. Engineering Analysis with Boundary Elements, 33(8-9), pp. 1100-1102, 2009.

https://doi.org/10.1016/j.enganabound.2009.02.005

[13] Johnson, A.N. \& Hromadka, T., Modeling mixed boundary conditions in a Hilbert space with the complex variable boundary element method (CVBEM). MethodsX, 2, pp. 292-305, 2015.

https://doi.org/10.1016/j.mex.2015.05.005 